\title{
Perfil de suscetibilidade antimicrobiana e presença do gene vapA em Rhodococcus equi de origem humana, ambiental e equina ${ }^{1}$
}

\author{
Lilian Kolling Girardini² ${ }^{2}$, Letícia Trevisan Gressler ${ }^{3}$, Mateus Matiuzzi da Costa ${ }^{4}$, Sônia \\ de Avila Botton ${ }^{3}$, Débora da Cruz Payão Pellegrini ${ }^{2}$ e Agueda Castagna de Vargas ${ }^{3 *}$
}

\begin{abstract}
Girardini L.K., Gressler L.T., Costa M.M., Botton S.A., Pellegrini D.C.P. \& Vargas A.C. 2013. [Susceptibility profile of Brazilian Rhodococcus equi isolates to different antimicrobial classes and the presence of vapA gene.] Perfil de suscetibilidade antimicrobiana e presença do gene vapA em Rhodococcus equi de origem humana, ambiental e equina. Pesquisa Veterinária Brasileira 33(6):735-740. Departamento de Medicina Veterinária Preventiva, Centro de Ciências Rurais, Universidade Federal de Santa Maria, Camobi, Santa Maria, RS 97105-900, Brazil. E-mail: agueda@ccr.ufsm.br

Rhodococcus equi is a facultative intracellular bacterium and etiological agent of rodococosis, an important disease that affects specially foals under six months old and leading the death generally due to pulmonary lesions. $R$. equi also has zoonotic potential, and it has emerged as an opportunistic pathogen in the world, specially infecting solid organ transplant recipients and immunocompromised human patients, mainly those infected by human immunodeficiency virus (HIV). Additionally, R. equi infections by healthy hosts have been reported principally in children and elderly individuals. Studies have shown an increasing level of resistance of isolates of $R$. equi against antibiotics commonly used to treat affected animals and humans. The virulence of this agent is associated with a protein located on a plasmid, designated vapA, it is essential for survival and replication of $R$. equi within macrophages. The present study evaluated the susceptibility profile of R.equi isolates from different sources against the antimicrobials most commonly used to treat animals and humans, as well as the occurrence and association of vapA gene and the antimicrobial multiple resistance index (AMRI). Sixty-seven Brazilian isolates of $R$. equi from different sources were analyzed: 30 clinical samples of horses, seven of human and 30 of environmental (six from soil and 24 from horse feces). To evaluate the susceptibility profile of R. equi isolates, the Kirby Bauer method was performed by using 16 drugs of 11 distinct antimicrobials classes. Additionally, those samples were also resistant to macrolides (azithromycin 6.7\%, erythromycin 6\% and clarithromycin 3.3\%) as well as rifamycin (13\%). All human and environmental samples were sensitive to macrolides and rifamycin. However, environmental isolates demonstrated high levels of resistance to penicillin and chloramphenicol. Similarly, human isolates had high level of resistance to ceftiofur, lincomycin and sulfazotrim. AMRI in all R. equi isolates ranged 0 to 0.67 , in clinical samples of horses the AMRI mean value obtained was 0.19 , in environmental was 0.14 and in human isolates was 0.1. Despite of high sensitivity observed in most Brazilian R. equi isolates analyzed, it was verified in clinical samples of horses different levels of resistance against all antibiotics commonly used in the treatment of rodococosis. In contrast, environmental isolates not demonstrated any resistance against antimicrobials employed in equine rodococosis therapy. In addition, in human isolates it was not observed resistance against drugs for
\end{abstract}

\footnotetext{
${ }^{1}$ Recebido em 26 de fevereiro de 2013.

Aceito para publicação em 4 de maio de 2013.

${ }^{2}$ Universidade do Oeste de Santa Catarina (Unoesc), Campus Xanxerê, Rodovia Rovilho Bortoluzzi, SC $480 \mathrm{Km} \mathrm{3,5,} \mathrm{Barro} \mathrm{Preto,} \mathrm{Xanxerê,} \mathrm{SC}$ 89820-000, Brasil.
}

\footnotetext{
${ }^{3}$ Depto Medicina Veterinária Preventiva (DMVP), Centro de Ciências Rurais (CCR), Universidade Federal de Santa Maria (UFSM), Santa Maria, RS 97105-900, Brasil. *Autor para correspondência: agueda@ccr.ufsm.br

${ }^{4}$ Colegiado de Zootecnia, Universidade Federal do Vale do São Francisco (Univasf), Rua José de Sá Maniçoba, Centro, Petrolina, PE 56306-410, Brasil.
} 
restricted use in human rodococosis therapy. Based on the AMRI achieved in clinical horse isolates, we highlight the importance of restrictive measures and more caution to use antimicrobial drugs in $R$. equi infections to avoid increasing of new multidrug-resistant strains.

INDEX TERMS: Rodococcosis, Rhodococcus equi, Equidae, bacterial resistance, AMRI.

RESUMO-- Rhodococcus equi é um micro-organismo intracelular facultativo, agente etiológico da rodococose, uma importante enfermidade que acomete principalmente potros com menos de seis meses de idade, causando a morte geralmente em decorrência de lesões pulmonares. Este agente também tem potencial zoonótico e emergiu como um patógeno oportunista no mundo, acometendo humanos imunocomprometidos, especialmente os transplantados e infectados pelo vírus da imunodeficiência humana (HIV). Entretanto, infecções por $R$. equi em hospedeiros hígidos tem sido relatadas, principalmente em crianças e idosos. Estudos tem mostrado um nível crescente na resistência de isolados de R. equi em relação aos antimicrobianos comumente utilizados no tratamento de animais e seres humanos infectados por este agente. A virulência deste pode estar associada a fatores como a cápsula de polissacarídeo, fosfolipase $\mathrm{C}$ e à enzima colesterol oxidase (fator equi). No entanto, uma proteína localizada em um plasmídeo, designada vapA, é essencial para a sobrevivência e replicação do agente em macrófagos. Com isso, os objetivos deste estudo foram avaliar o perfil de suscetibilidade de isolados de $R$. equi de diferentes fontes em relação aos antimicrobianos mais comumente utilizados na terapêutica animal e humana, bem como verificar a associação entre a presença do gene vapA e o índice de resistência múltipla aos antimicrobianos (IRMA). Neste estudo, 67 isolados brasileiros de $R$. qui de diferentes fontes foram analisados: 30 provenientes de amostras clínicas de equinos, sete de humanos e 30 ambientais (seis do solo e 24 de fezes de equinos). Para avaliar o perfil de suscetibilidade dos isolados utilizou-se o método de disco difusão, sendo testadas 16 drogas de diferentes classes de antimicrobianos. As amostras clínicas de equinos apresentaram as maiores taxas de resistência à penicilina $(86,7 \%)$ e lincomicina (30\%). Além disso, foram também resistentes a macrolídeos (azitromicina a 6,7\%, eritromicina a $6 \%$ e claritromicina a 3,3\%) e rifamicina (13\%). Todas as amostras humanas e ambientais foram sensíveis aos macrolídeos e rifamicina. Contudo, isolados ambientais demonstraram níveis elevados de resistência à penicilina e cloranfenicol. Da mesma forma, os isolados humanos apresentaram alto nível de resistência ao ceftiofur, lincomicina e sulfazotrim. O IRMA em todos os isolados de R. equi variou de 0 a 0,67 , tendo como valores médios 0,19 para as amostras clínicas de equinos, 0,14 nas ambientais e em isolados humanos foi de 0,1. Apesar da alta sensibilidade observada nos isolados analisados, verificaram-se diferentes níveis de resistência nas amostras clínicas de equinos. Em contraste, os isolados ambientais não demonstraram resistência em relação aos agentes antimicrobianos utilizados na terapia da rodococose equina. Além disso, em isolados humanos não se observou resistência contra a droga para uso restrito em terapia de humano. Com base no IRMA observado em isolados clínicos de equinos, destacamos a importância de medidas restritivas e mais cautela na utilização de antimicrobianos em infecções causadas por $R$. equi para evitar o aumento de novas cepas multirresistentes.

TERMOS DE INDEXAÇÃ̃: Rodococose, Rhodococcus equi, equinos, resistência bacteriana, IRMA.

\section{INTRODUÇÃO}

Rhodococcus equi é um dos micro-organismos mais importantes em afecções pulmonares de potros com idade inferior a seis meses de idade (Linder 1997, Monego et al. 2009), normalmente relacionados a quadros de broncopneumonia piogranulomatosa crônica e enterite associada à linfadenite (Prescott 1991). Além disso, tem sido considerado um patógeno oportunista emergente tanto para humanos imunossuprimidos, quanto para indivíduos hígidos, especialmente crianças e idosos (Kedlaya et al. 2001).

A virulência de R. equi está associada a fatores como uma cápsula polissacarídica, e às enzimas fosfolipase $\mathrm{C}$ e colesterol oxidase (fator equi). Entretanto, a presença da cápsula não é essencial para a sobrevivência do agente no ambiente (Sydor et al. 2008) ao mesmo tempo que cepas avirulentas podem secretar enzimas do "fator equi" (Pei et al. 2006). 0 fator mais relevante associado à sua patogenicidade é uma proteína codificada por um gene plasmidial denominada vapA ("virulence associated protein") (Takai et al. 1991, Sekizaki et al. 1995). A expressão da vapA é fundamental para a prevenção da acidificação do fagossomo, o que favorece a sobrevivência e replicação do micro-organismo no interior de macrófagos (Von Barden \& Haas 2009).

O tratamento das infecções por $R$. equi é realizado com antimicrobianos lipofílicos durante um período prolongado (Prescott 1991), utilizando-se a combinação de macrolídeos como a eritromicina associados à rifampicina (Giguère \& Prescott 1997). No entanto, o surgimento de cepas resistentes tem sido relatado nas últimas décadas (Nordman \& Ronco 1992, Takai et al. 1997, Asoh et al. 2003, Niwa et al. 2006, Buckley et al. 2007, Venner et al. 2012) Desse modo, este estudo teve como objetivos avaliar o perfil de suscetibilidade de isolados de R. equi de diferentes origens frente aos principais antimicrobianos utilizados no tratamento de animais e humanos e verificar a associação entre a presença do gene vapA e o índice de resistência múltipla aos antimicrobianos (IRMA).

\section{MATERIAL E MÉTODOS}

Sessenta e sete cepas brasileiras de Rhodococcus equi, isoladas entre os 1993 e 2008 foram estudadas, sendo 30 amostras clínicas de equinos provenientes de diversos tecidos (fragmentos de pulmão, linfonodos e glândula mamária), secreções (lavado traqueal, líquidos abdominal e sinovial, abscessos subcutâneo e inguinal) e fezes coletadas diretamente da ampola retal de um animal com 
diarréia; sete amostras de humanos oriundas de pacientes hígidos do Rio Grande do Sul e de casos clínicos de pacientes do estado do Rio de Janeiro; além de 30 amostras ambientais provenientes de solo e fezes. Os isolados foram previamente classificados em gênero e espécie de acordo com características fenotípicas (Lazzari et al. 1997, Monego et al. 2009). Todos os isolados de R. equi permaneceram armazenados liofilizados a $-20^{\circ} \mathrm{C}$ até a realização dos testes laboratoriais. Para confirmação da identidade dos isolados, bem como classificação em relação à presença do gene de virulência vapA, realizou-se a técnica de PCR, utilizando-se como controle positivo uma cepa previamente sequenciada, segundo técnica descrita por Monego et al. (2009).

A avaliação da suscetibilidade antimicrobiana in vitro foi realizada pelo método Kirby Bauer modificado (CLSI 2003, CLSI 2008). Testaram-se as seguintes classes e respectivos antimicrobianos: macrolídeos - azitromicina $(15 \mu \mathrm{g})$, claritromicina $(15 \mu \mathrm{g})$ e eritromicina $(15 \mu \mathrm{g})$; aminoglicosídeos - gentamicina $(10 \mu \mathrm{g})$; quinolo-

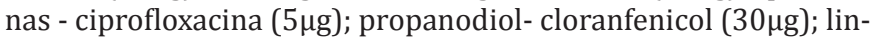
cosamidas - lincomicina $(2 \mu \mathrm{g})$; oxazolidinonas - linezolida $(30 \mu \mathrm{g})$; glicopeptídeos - vancomicina $(30 \mu \mathrm{g})$; beta-lactâmicos- penicilina (10 UI), imipenem $(10 \mu \mathrm{g})$ e ceftiofur $(30 \mu \mathrm{g})$; polimixinas - polimixina B (300 UI); rifamicinas - rifampicina ( $5 \mu \mathrm{g})$; sulfamidas - sulfazotrim $(25 \mu \mathrm{g})$ e tetraciclinas - tetraciclina $(30 \mu \mathrm{g})$.

0 índice de resistência múltipla aos antimicrobianos (IRMA) foi calculado conforme a metodologia descrita por Krumperman (1983), sendo determinado pela relação entre o número de classes antimicrobianas para as quais a amostra é resistente e o número total de diferentes classes antimicrobianas testadas. Posteriormente, calculou-se o valor médio do IRMA em função das diferentes origens dos isolados (clínica de equinos, ambiental, humana). Sugere-se que valores acima de 0,2 são considerados indicadores da presença de genes de resistência aos antimicrobianos, adotando-se, portanto este padrão (Krumperman 1983).

Os dados foram analisados pelo teste t ao nível de significância de 5\%, no programa estatístico SPSS para Windows versão 18.0, a fim de avaliar estatisticamente a relação entre os isolados com e sem a presença de vapA quanto ao IRMA apresentado.

\section{RESULTADOS E DISCUSSÃO}

A maioria dos isolados brasileiros de Rhodococcus equi estudados apresentaram perfil de suscetibilidade frente aos diferentes antimicrobianos testados. Em relação ao teste de suscetibilidade aos antimicrobianos, foi possível observar, em $100 \%$ das amostras analisadas $(n=67)$, sensibilidade ao imipenem e gentamicina. Entretanto, os aminoglicosídeos apresentam baixa penetração nos macrófagos ou neutrófilos; além de ser relatada a ocorrência de efeitos adversos, como a nefrotoxicidade, o que restringe sua aplicação em equinos (Guardabassi et al. 2008).

Apenas oito cepas foram sensíveis a todos os antimicrobianos testados, sendo quatro ambientais, três clínicas e uma humana. Vinte e seis isolados de origem ambiental (86,7\%), 27 provenientes de equinos $(84,4 \%)$ e cinco de humanos $(71,4 \%)$ demonstraram resistência à penicilina. Como os perfis de multirresistência foram definidos a partir dos resultados obtidos em um teste de suscetibilidade fenotípico, considerou-se como multirresistente apenas os isolados que apresentaram resistência a três ou mais classes de antimicrobianos (Schwarz et al. 2010). Desse modo, dos 30 isolados ambientais, 30 de equinos e sete de origem humana analisados, nove, 13 e três apresentaram perfil de multirresistência, respectivamente. Os perfis multirresis-

\section{Quadro 1. Perfis de multirresistência a antimicrobianos apresentados pelos isolados de Rhodococcus equi de origem ambiental, equinos e humanos no Brasil}

\begin{tabular}{clc}
\hline $\begin{array}{c}\text { Origem dos } \\
\text { isolados }\end{array}$ & \multicolumn{1}{c}{$\begin{array}{c}\text { Perfis de multirresistência } \\
\text { aos antimicrobianos }\end{array}$} & $\begin{array}{c}\text { No de } \\
\text { isolados }\end{array}$ \\
\hline Ambientais & PEN/VAN/CLO/ERI/LIN/LIZ & 1 \\
& PEN/CLO/LIN/SUT/POL B & 1 \\
& PEN/CLO/SUT/POL B & 1 \\
& PEN/TET/CLO/LIN & 1 \\
& PEN/CLO/LIN & 1 \\
& PEN/CLO/POL B & 3 \\
& CTF/CIP/SUT & 1 \\
Equinos & PEN/VAN/TET/CLA/ERI/LIN/RIF/LIZ/SUT & 1 \\
& PEN/VAN/TET/CLO/CLA/ERI/LIN/RIF/LIZ & 1 \\
& CTF/VAN/CLO/RIF/CIP/POL B & 1 \\
& PEN/VAN/ERI/LIN/RIF/LIZ & 1 \\
& PEN/TET/CLO/LIN/POL B & 1 \\
& PEN/VAN/CLO/LIN/LIZ & 1 \\
& PEN/TET/LIN/POL B & 1 \\
& PEN/LIN/POL B & 1 \\
& CLO/AZI/SUT & 1 \\
& CTF/CLO/POL B & 1 \\
& CTF/LIN/POL B & 2 \\
& CTF/LIN/SUT & 1 \\
CTF/LIN/SUT/POL B & 1 \\
Humanos & 1 \\
& CTF/LIN/RIF & 1
\end{tabular}

$\overline{\mathrm{CTF}}=$ ceftiofur, $\mathrm{PEN}=$ penicilina, $\mathrm{IM} \mathrm{P}=$ imipenem, $\mathrm{GEN}=$ gentamicina, VAN = vancomicina, TET $=$ tetraciclina, $\mathrm{CLO}=$ cloranfenicol, $\mathrm{CLA}=$ clari tromicina, $\mathrm{ERI}=$ eritromicina, $\mathrm{AZI}=$ azitromicina, $\mathrm{LIN}=$ lincomicina, $\mathrm{RIF}$ = rifampicina, $\mathrm{CIP}=$ ciprofloxacina, $\mathrm{LIZ}=$ linezolida, $\mathrm{SUT}=$ sulfazotrin, $\mathrm{POL}=$ Polimixina $\mathrm{B}$.

tência encontram-se discriminados no quadro 1. Quanto aos fármacos ceftiofur e sulfazotrim foram observados menores índices de sensibilidade nos isolados humanos. Já os isolados ambientais apresentaram menores índices de sensibilidade frente ao cloranfenicol. Todavia, o perfil de sensibilidade à vancomicina foi variável quanto à origem dos isolados; onde os isolados oriundos de humanos apresentaram $100 \%$ de sensibilidade, seguidos dos isolados ambientais (97\%) e de equinos (84\%). Da mesma forma, em relação à polimixina $\mathrm{B}$, isolados humanos apresentaram-se $100 \%$ sensíveis, enquanto os isolados clínicos de equinos e ambientais apresentaram índices de 80\% e 90\% de sensibilidade, respectivamente.

As amostras provenientes de equinos apresentaram perfil de suscetibilidade à claritromicina, eritromicina e azitromicina, com índices de resistência de apenas 3,3\%, $6 \%$ e $6,7 \%$, respectivamente. Entretanto, observou-se $13 \%$ de resistência frente à rifampicina. Nas amostras humanas e ambientais observou-se $100 \%$ de sensibilidade para os antimicrobianos da classe dos macrolídeos e à rifampicina (Fig.1). Os dados de sensibilidade à rifampicina diferem dos encontrados em outro estudo (Nordmann et al. 1992), no qual se observou a ocorrência de resistência. No entanto, a alta sensibilidade encontrada neste trabalho pode estar relacionada ao pequeno número de amostras humanas avaliadas. Embora a maioria dos isolados brasileiros de $R$. equi estudados apresentaram perfil de suscetibilidade frente aos diferentes antimicrobianos testados, as amostras clínicas de equinos revelaram um expressivo índice de resistência à rifampicina e, em menor proporção, à eritro- 

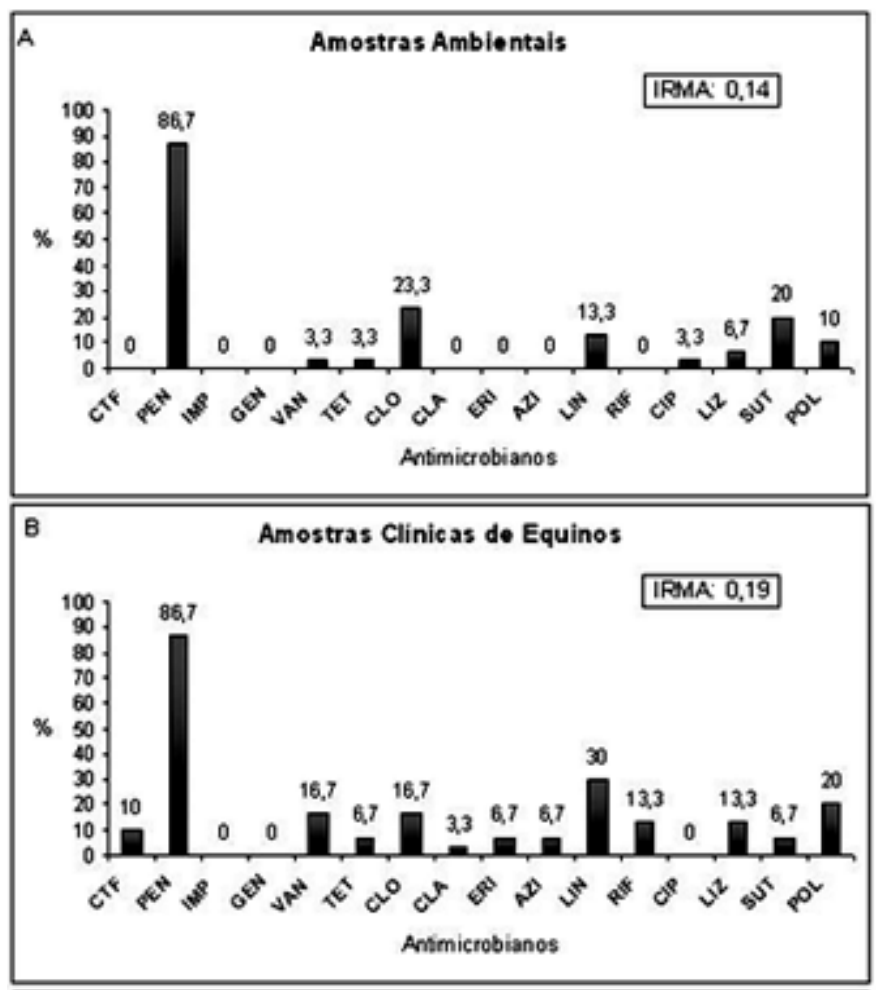

C Amostras Humanas

IRMA: 0.1

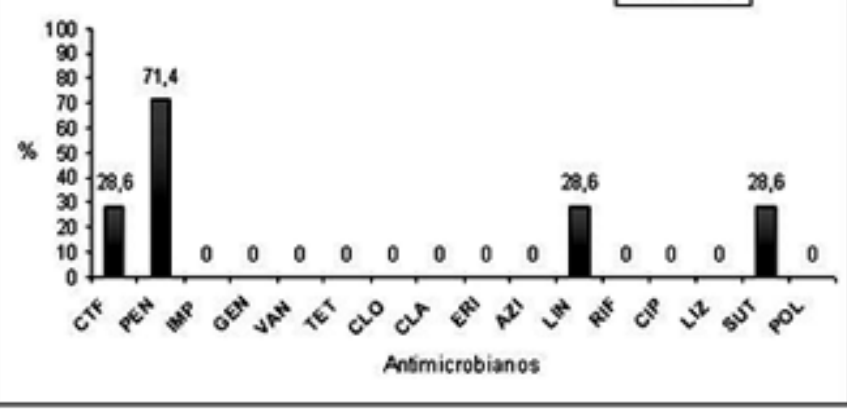

Fig.1. (A) Perfil de suscetibilidade de isolados de Rhodococcus equi de amostras ambientais, (B) amostras clínicas de equinos e (C) amostras humanas frente a 16 antimicrobianos, sendo $\mathrm{CTF}=$ ceftiofur, PEN = penicilina, IMP = imipenem, GEN = gentamicina, VAN = vancomicina, TET $=$ tetraciclina, CLO $=$ cloranfenicol, $\mathrm{CLA}=$ claritromicina, $\mathrm{ERI}=$ eritromicina, $\mathrm{AZI}=$ azitromicina, LIN = lincomicina, RIF = rifampicina, CIP = ciprofloxacina, $\mathrm{LIZ}=$ linezolida, $\mathrm{SUT}=$ sulfazotrin, $\mathrm{POL}=$ Polimixina $\mathrm{B} . \mathrm{IRMA}=$ Índice de Resistência Múltipla aos Antimicrobianos.

micina. Estes dados indicam a possibilidade dos isolados terem sofrido uma maior pressão de seleção devido a utilização destes antimicrobianos (Guardabassi et al. 2008), uma vez que, a partir da década de 1980 o tratamento preconizado nos casos de rodococose equina passou a ser a associação de rifampicina e eritromicina (Prescott \& Baggot 1993).

Além disso, a eritromicina apresenta absorção variável quando administrada oralmente, o que pode comprometer ainda mais a eficácia no tratamento de infecções causadas por este agente (Jacks et al. 2003). 0 crescimento no número de isolados apresentando resistência a rifampicina e eritromicina foi descrito em um estudo com cepas de R. equi isoladas durante 10 anos, onde foi possível verificar que $4 \%$ destas apresentaram resistência a estes antimicrobianos (Giguére et al. 2010). Desta forma, ressalta-se a importância de estudos que avaliem a ação de diferentes agentes antimicrobianos como alternativa em casos de resistência a esses fármacos (Javsicas et al. 2010).

Índices expressivos de resistência à lincomicina foram observados em isolados clínicos de equinos (30\%) e humanos (28\%). Além disso, a utilização de lincomicina em equinos é restrita devido ao alto risco da ocorrência de colite fatal (Prescott 1991). Com exceção dos isolados ambientais, não se observou resistência frente à ciprofloxacina. No entanto, a utilização deste fármaco torna-se restrita devido à possibilidade de ocorrência de danos articulares associados ao uso de quinolonas em potros (López et al. 2000)

Em relação à linezolida, os isolados clínicos de equinos apresentaram $13,3 \%$ de resistência, enquanto os isolados ambientais apenas $3 \%$ e as amostras humanas foram $100 \%$ sensíveis. Conforme Bowersock et al. (2000) este fármaco apresenta bons resultados de sensibilidade e é considerado eficiente no tratamento tanto de humanos quanto de animais. Todavia, as oxazolidinonas não devem ser empregadas na medicina veterinária, resguardando-as para utilização na medicina humana (Bowersock et al. 2000).

Com relação ao IRMA observou-se índice acima de 0,2 em $26 \%$ dos isolados clínicos de equinos e $16 \%$ dos isolados ambientais. Em isolados humanos não se observou IRMA acima de 0,2, sendo encontrado o valor médio de 0,1. As amostras ambientais obtiveram valor médio de IRMA de 0,14 , enquanto que nas amostras clínicas de equinos o valor médio encontrado foi 0,19 , variando de 0 a 0,67 (Fig.1). Elevados valores de IRMAs podem estar associados a falhas no tratamento de enfermidades em animais e humanos (Mota et al. 2005). Os IRMASs observados nos isolados clínicos de equinos apontam para um perfil de multirresistência, diferentemente dos isolados ambientais, reforçando as evidências de que insucessos no tratamento contribuam para a seleção de R. equi resistentes aos antimicrobianos preconizados no tratamento. 0 desenvolvimento da resistência bacteriana tem sido atribuído em parte ao uso de doses terapêuticas que frequentemente bloqueiam o crescimento de células bacterianas suscetíveis. Em algumas condições, a concentração pode ser tão baixa ao ponto de eliminar apenas um pequeno número de mutantes resistentes a drogas na população infectada, recebendo a denominação de janela de seleção de mutantes (mutant selection window - MSW). 0 limite superior à MSW denominado mutant prevention concentration (MPC) consiste na concentração antimicrobiana capaz de inibir o crescimento de subpopulações resistentes aos antimicrobianos devido à mutação espontânea. Estudos indicam que concentrações de antimicrobianos acima da MPC como terapia podem reduzir a chance de surgimento de mutantes resistentes e elevados níveis de resistência (Drlica 2003, Drlica \& Zhao 2007).

Dentre todas as amostras avaliadas quanto à presença do gene vapA, somente as amostras clínicas provenientes de equinos $(n=30)$ apresentaram resultado positivo pela técnica de PCR. A presença do gene vapA nas amostras clí- 
nicas de equinos concordam com os dados encontrados no estudo realizado por Ribeiro et al. (2005), reforçando a importância da vapA na patogênese das enfermidades causadas por R. equi. A vapA é necessária para o crescimento bacteriano intracelular em macrófagos (Jain et al. 2003), mas, não é suficiente para a virulência bacteriana. Um estudo demonstrou que após a cura de um plasmídeo derivado de isolado bacteriano virulento, este não foi capaz de restaurar a virulência em camundongos e potros, indicando que genes determinantes de virulência além do vapA podem residir em plasmídeos (Ren \& Prescott 2004). Em média, as amostras com a presença do gene vapA tiveram maiores valores de IRMA (M=0,1867; $\mathrm{EP}=0,2871)$ quando comparadas às amostras onde não foi possível detectar a presença deste mesmo gene $(M=0,137 ; \mathrm{EP}=0,01765)$. Entretanto, esta diferença não foi significativa em nível de $5 \%$ $(\mathrm{p}=0,131)$. Provavelmente os isolados clínicos positivos para o gene vapA, estão mais sujeitos a uma maior pressão de seleção pela utilização de antimicrobianos para tratamento das afecções clínicas (Drlica 2003, Drlica \& Zhao 2007). Nesse sentido, testes de suscetibilidade aos antimicrobianos devem ser empregados na prática clínica e rotina laboratorial, uma vez que fornecem subsídios para a adoção de medidas terapêuticas adequadas (Schwalbe et al. 2007, Guardabassi et al. 2008).

Por fim, ressalta-se a necessidade da adoção de medidas restritivas e maior cautela no emprego destes fármacos na rotina terapêutica veterinária das infecções por este patógeno, a fim de evitar a emergência de novas cepas de $R$. equi multirresistentes.

\section{CONCLUSÕES}

0 perfil de susceptibilidade dos isolados brasileiros de Rhodococcus equi frente às diferentes classes de antimicrobianos analisadas evidencia alta sensibilidade da maioria dos isolados testados.

As amostras clínicas de equinos apresentaram diferentes graus de resistência às drogas utilizadas no tratamento de rotina para rodococose equina.

As amostras clínicas de equinos apresentaram o gene $v a p A$ e IRMA mais elevado quando comparadas às amostras negativas para o mesmo gene.

Declaração de interesse.- Os autores relatam não haver nenhum conflito de interesse e são os únicos responsáveis pelo conteúdo e pela redação do trabalho.

\section{REFERÊNCIAS}

Asoh N., Watanabe H., Fines-Guyon M., Watanabe K., Oishi K., Kositsakulchai W., Sanchai T., Kunsuikmengrai K., Kahintapong S., Khantawa B., Tharavichitkul P., Sirisanthana T. \& Nagatake T. 2003. Emergence of rifampin-resistant Rhodococcus equi with several types of mutations in the rpoB gene among AIDS patients in northern Thailand. J. Clin. Microbiol. 41:2337-2340.

Bowersock T.L., Salmon S.A., Portis E.S., Prescott J.F., Robison D.A., Ford C.W. \& Watts J.L. 2000. MICs of Oxazolidinones for Rhodococcus equi Strains Isolated from Humans and Animals. Antimicrob. Agents Chemother. 44:1367-1369.

Buckley T., McManamon E. \& Stanbridge S. 2007. Resistance studies of erythromycin and rifampin for Rhodococcus equi over a 10-year period. Irish Vet. J. 60(12):728-731.
CLSI 2003. Performance standards for antimicrobial disk susceptibility tests. Clinical and Laboratory Standards Institute. Approved Standard. $8^{\text {th }}$ ed. CLSI/NCCLS document M2 - A8. Wayne.

CLSI 2008. Performance standards for antimicrobial disk and dilution susceptibility tests for bacteria isolated from animals. Clinical and Laboratory Standards Institute. Approved Standard, $3^{\text {rd }}$ ed. CLSI/NCCLS document M31 - A3. Wayne.

Drlica K. 2003. The mutant selection window and antimicrobial resistance. J. Antimicrob. Chemother. 52:11-17.

Drlica K. \& Zhao X. 2007. Mutant selection window hypothesis updated. Clin. Infect. Dis. 44:681-688.

Giguère S. \& Prescott J.F. 1997. Clinical manifestations, diagnosis, treatment, and prevention of Rhodococcus equi infections in foals. Vet. Microbiol. 56:313-334.

Giguère S., Hondalus M.K., Yager J.A., Darrah P., Mosser D.M. \& Prescott J. F. 1999. Role of the $85 \mathrm{~Kb}$ plasmid and plasmid encoded virulence protein A in intracellular survival and virulence of Rhodococcus equi. Infect Immun. 67:3548-3557.

Giguére S., Lee E., Williams E., Cohen N.D., Chaffin M.K., Halbert N., Martens R.J., Franklin R.P., Clark C.C. \& Slovis N.M. 2010. Determination of the prevalence of antimicrobial resistance to macrolide antimicrobials or rifampin in Rhodococcus equi isolates and treatment outcome in foals infected with antimicrobial-resistant isolates of R. equi. J. Am. Vet. Med. Assoc. 237:74-81.

Guardabassi L., Jensen L.B. \& Kruse H. 2008. Guide to Antimicrobial Use in Animals. Blackwell Publishing, Oxford. 223p.

Jacks S.S., Giguère S. \& Nguyen A. 2003. In vitro susceptibilities of Rhodococcus equi and other common equine pathogens to azithromycin, clarithromycin, and 20 other antimicrobials. Antimicrob. Agents Chemother. 47:1742-1745.

Jain S., Bloom B.R. \& Hondalus M.K. 2003. Deletion of vapA encoding virulence associated protein $\mathrm{A}$ attenuates the intracellular actinomycete Rhodococcus equi. Mol. Microbiol. 50:115-128.

Javsicas L.H., Giguère S. \& Womble A.Y. 2010. Disposition of oral telithromycin in foals and in vitro activity of the drug against macrolide-susceptible and macrolide-resistant Rhodococcus equi isolates. J. Vet. Pharmacol. Ther. 33:383-388.

Kedlaya I., Ing M.B. \& Wong S.S. 2001. R. equi infections in immunocompetent hosts: case report and review. Clin. Infect. Dis. 32:39-47.

Krumperman P.H. 1983. Multiple antibiotic resistance indexing of Escherichia coli to identify high-risk sources of fecal contamination of foods. Appl. Environ. Microbiol. 46:165-170.

Lazzari A., Vargas A.C., Dutra V., Costa M.M. \& Flores L.A.S. 1997. Aspectos epidemiológicos do Rhodococcus equi em equinos do município de Bagé, RS, Brasil. Ciência Rural 27:441-446.

Linder R. 1997. Rhodococcus equi and Arcanobacterium haemolyticum: two "Coryneform" bacteria increasingly recognized as agents of human infection. EID 3:1-10.

López H.S., Cambreros L.O., Madrigal I.L. \& Jurgens K.O. 2000. Reacciones adversas de los fármacos en los eqüinos. Veterinaria México 31(4):329354.

Monego F., Maboni F., Krewer C., Vargas A.C., Costa M. \& Loreto E. 2009. Molecular characterization of Rhodococcus equi from Horse-Breeding Farms by means of multiplex PCR for the vap gene family. Curr. Microbiol. 58:399-403.

Mota R.A., Silva K.P.C., Freitas M.F.L., Porto W.J.N. \& Silva L.B.G. 2005. Utilização indiscriminada de antimicrobianos e sua contribuição a multirresistência bacteriana. Braz. J. Vet. Res. Anim. Sci. 42: 465-470.

Niwa H., Hobo S. \& Anzai T. 2006. A nucleotide mutation associated with fluoroquinolone resistance observed in gyrA of in vitro obtained Rhodococcus equi mutants. Vet. Microbiol.115:264-268.

Nordmam P. \& Ronco E. 1992. In-vitro antimicrobial suscetibility of Rhodococcus equi. J. Antimicrob. Chemotherapy 29(4):383-393.

Nordmam P., Rouveix E., Guenounou M. \& Nicolas M.H. 1992. Pulmonary 
abscess due to a rifampin and fluoroquinolone resistant Rhodococcus equi strain in a HIV infected patient. Eur. J. Clin. Microbiol. Infect. Dis. 11:557-558

Pei Y., Dupont C., Sydor T., Haas A. \& Prescott J.F. 2006. Cholesterol oxidase (ChoE) is not important in the virulence of Rhodococcus equi. Vet. Microbiol. 118:240-246.

Prescott J.F. 1991. Rhodococcus equi: an animal and human pathogen. Clin. Microbiol. Rev. 4:20-34.

Prescott J.F. \& Baggot J.D. 1993. Antimicrobial Therapy in Veterinary Medicine. $2^{\text {nd }}$ ed. University Press, Iowa. 612p.

Ren J. \& Prescott J.F. 2004. The effect of mutation on Rhodococcus equi virulence plasmid gene expression and mouse virulence. Vet. Microbiol. 103:219-230.

Ribeiro M.G., Seki I., Yasuoka K., Kakuda T., Sasaki Y., Tsubaki S. \& Takai S. 2005. Molecular epidemiology of virulent Rhodococcus equi from foals in Brazil: virulence plasmids of 85-kb type I, 87-kb type I, and a new variant, 87-kb type III. Comp. Immunol. Microbiol. Infect. Dis. 28:5361.

Schwalbe R., Steele-Moore L. \& Goodwin A.C. 2007. Antimicrobial Susceptibility Testing Protocols. CRC Press, New York. 414p.

Schwarz S., Silley P., Woodford N., Van Dijikeren E., Johnson A.P. \& Gaastra
W. 2010. Assessing the antimicrobial susceptibility of bacteria obtained from animals. J. Antimicrob. Chemother. 65:601-604.

Sekizaki T., Takai S., Egawa Y., Ikeda T., Ito H. \& Tsubaki S. 1995. Sequence of the Rhodococcus equi gene encoding the virulence-associated 15-17kDa antigens. Gene 155:135-136.

Sydor T., Von Bargen K., Becken U., Spuerck S., Nicholson V.M., Prescott J.F. \& Haas A. 2008. A mycolyl transferase mutant of Rhodococcus equi lacking capsule integrity is fully virulent. Vet. Microbiol. 128:327-341.

Takai S., Sekizaki T., Ozawa T., Sugawara T., Watanabe Y. \& Tsubaki S. 1991. Association between a large plasmid and 15- to 17-kilodalton antigens in virulent Rhodococcus equi. Infect. Immun. 59:4056-4060.

Takai S., Takeda K., Nakano Y., Karasawa T., Furugoori J., Sasaki Y., Tsubaki S., Higuchi T., Anzai T., Wada R. \& Kamada M. 1997. Emergence of rifampicin-resistant Rhodococcus equi in an infected foal. J. Clinical Microbiol. 35:1904-1908.

Venner M., Rödiger A., Laemmer M. \& Giguère S. 2012. Failure of antimicrobial therapy to accelerate spontaneous healing of subclinical pulmonary abscesses on a farm with endemic infections caused by Rhodococcus equi. Vet. J. 192(3):293-298.

Von Bargen K. \& Haas A. 2009. Molecular and infection biology of the horse pathogen Rhodococcus equi. FEMS Microbiol. Rev. 33:870-891. 